\title{
Endoglucanase activity of novel Aspergillus sydowii isolate WIU-01 and application for hydrophyte degradation
}

\author{
Zhang H.P. ${ }^{1,2}$, Li Q.Z. ${ }^{2,3}$, Zhou Q.H. ${ }^{2}$ and Wu Z.B. ${ }^{2 *}$ \\ ${ }^{1}$ College of Resources and Environmental Engineering, Wuhan University of Technology, Wuhan 430070, P. R. China \\ ${ }^{2}$ State Key Laboratory of Freshwater Ecology and Biotechnology, Institute of Hydrobiology, Chinese Academy of Sciences, Wuhan \\ 430072, P. R. China \\ ${ }^{3}$ College of Advanced Agricultural Sciences, University of Chinese Academy of Sciences, Beijing 100049, P. R. China \\ Received: 26/05/2021, Accepted: 24/07/2021, Available online: 15/08/2021 \\ *to whom all correspondence should be addressed: e-mail: wuzb@ihb.ac.cn \\ https://doi.org/10.30955/gnj.003744
}

\section{Graphical abstract}
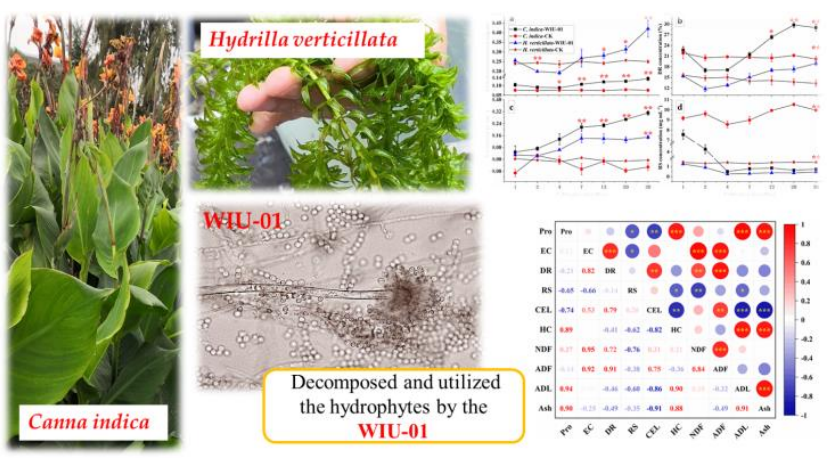

\section{Abstract}

Many eutrophic lakes contain rapidly growing hydrophytes. Overgrown biomass is usually mechanically harvested and thrown away, leading to resource waste and secondary environmental pollution. Microbial degradation is an economically and environmentally friendly approach for managing hydrophytic waste, fuelling the search for efficient biomass degraders. Here, we present isolation and characterization of Aspergillus sydowii WIU-01, a novel cellulolytic fungus. Strain WIU-01 was isolated from air. The degradation rate (29.75 vs. $21.95 \%)$ and endoglucanase activity ( 0.31 vs. $0.16 \mathrm{U} \mathrm{mL}^{-1}$ ) of the fungus were higher in Canna indica (emergent plant) medium than in Hydrilla verticillata (submerged plant) medium, accordingly. Further, fungal endoglucanase activity was significantly positively correlated with the degradation rate, neutral detergent fiber content, and acid detergent fiber content of hydrophyte powder. Fungal biomass was significantly negatively correlated with reducing sugar and cellulose content of hydrophyte medium, but was significantly positively correlated with hemicellulose, acid detergent lignin, and ash content of the medium. Collectively, these observations indicate that $A$. sydowii decomposes emergent and submerged plant mass without acid-base sample pretreatment, albeit its endoglucanase activity is relatively low. This highlights the role of cellulolytic microorganisms in the natural environment and the notion that the environment can be a source of cellulolytic microorganisms for potential environmentally friendly applications.

Keywords: Cellulolytic fungus; endoglucanase; Canna indica; Hydrilla verticillata; fiber fraction.

\section{Introduction}

Hydrophytes are important biological resources in the aquatic ecosystem, as they play central role in the energyflow of aquatic material and ecosystem stability (Van Echelpoel and Goethals, 2018). However, increase in organic nutrient levels (e.g., from pollution, abusive discharge, or atmospheric deposition) or introduction of alien hydrophytes into the environment (e.g., water lily) can cause hydrophyte overgrowth (Zhao et al., 2012). The overgrowth usually leads to the accumulation of considerable biomass in some eutrophic lakes. Generally, aquatic plant overgrowth negatively impacts the ecosystem, e.g., by creating hypoxic conditions, crowding the water body, decreasing the light penetrance, and reducing the biodiversity (Geary and Kim, 2001). Further, the decaying hydrophyte residue may act as a secondary pollutant that is detrimental to nutrient elements, such as releasing the nitrogen and phosphorus, in the water body (Welsch and Yavitt, 2003). Consequently, the overgrown hydrophytes have to be effectively managed and utilized.

At present, hydrophytes are mainly used for the production of biological fertilizers, fuels, etc. (Kaur et al., 2014). As the biomass is a recalcitrant network of lignocellulose containing cellulose (CEL), hemicellulose (HC), and lignin, lignocellulose hydrolysis is the rate-limiting step of biomass utilization (Amezcua-Allieri et al., 2017). Consequently, the biomass has to be pretreated to disrupt lignocellulosic structure to enhance accessibility of CEL and other fiber fractions. CEL is a carbohydrate polymer composed of repetitive fibrils of $\beta$-D-glucopyranose linked by $\beta$-1,4glycosidic bonds (Sajab et al., 2019). Various physical, chemical, biological, and physicochemical methods are used for biomass pretreatment. The non-biological methods have serious limitations, such as high energy 
consumption, and generation of by-products or chemical waste. By contrast, biological pretreatment methods that utilize microbes for lignocellulose degradation are environmentally friendly and low-cost methods.

Synergistic activity of multiple enzymes greatly improves the depolymerization of CEL. Endoglucanases (EG) preferentially cleave the $\beta$-glucosidic bond, randomly hydrolyzed the amorphous regions of fibrils. Some microorganisms (bacteria, fungi, or yeasts) produce EG that convert CEL to soluble oligosaccharides (Mohapatra et al., 2018b). Filamentous fungi, such as Aspergillus spp., play a vital role in CEL degradation, as they produce an array of enzymes that decompose plant cell wall polysaccharides (CEL, HC, and pectin) (Kowalczyk et al., 2017). Aspergillus is an ancient organism with strong adaptive capacity, and is widely distributed in the biosphere (Jurjevic et al., 2012). In fact, many Aspergillus species have been studied from the perspective of plant biomass degradation, and possess good cellulase and $\beta$-glucosidase activity (Benoit et al., 2015, Makela et al., 2018, Raulo et al., 2016). However, in many cases, acid-base pretreatment of the plant biomass is required before microbial degradation and utilization, and this increases the processing cost. Accordingly, identification of new microbes that efficiently decompose and utilize plants without any pretreatment and in the natural environment draws considerable interest.

Some CEL decomposing microorganisms have been identified over the years. As early as in 1987, Bhat and Maheshwari (1987) studied the activity of components of the extracellular cellulase system of the thermophilic fungus isolated from the soil. Hernández et al. (2001) isolated Streptomyces sp. UAH 47 from lignocellulosic residues. Yoon and Kim (2005) showed that cellulases produced by the brown rot basidiomycete Fomitopsis palustris degrade crystalline CEL to soluble sugars. Further, Herve et al. (2014) reported on the white-rot fungus Phanerochaete chrysosporium screened from the beech forest soil for wood decomposition.

The above microbes have been isolated from an environment that contains CEL or decaying plants. However, we focused that the harvested plant leaves would be decayed and decomposed in the air environment. These microbes using CEL as a carbon source should survive dispersion in air. Therefore, it is also important to characterize air-borne CEL-decomposing microorganisms. As a proof of concept, we here isolated strains with crystalline CEL-degrading activity from air to prove that the presence of microbes in the air could cause the decomposition of plants. Moreover, we also characterized the hydrophyte-degrading ability of the most promising isolate to assess its future potential applications.

\section{Materials and methods}

\subsection{Isolation of CEL-degrading microorganisms from air}

Air-borne microbes with strong survival capacity were isolated in a primary screening medium, with sodium carboxymethyl CEL (CMC-Na) as the only carbon source. The medium also contained $\left(\mathrm{NH}_{4}\right)_{2} \mathrm{SO}_{4}, \mathrm{~K}_{2} \mathrm{HPO}_{4}$, $\mathrm{MgSO}_{4} \cdot 7 \mathrm{H}_{2} \mathrm{O}$, peptone, yeast extract, and agar $(2 \%, \mathrm{w} / \mathrm{v})$. The medium was autoclaved $\left(121{ }^{\circ} \mathrm{C}\right.$ for $\left.30 \mathrm{~min}\right)$, and poured into a sterile culture dish (10-cm in diameter). Next, an uncovered culture dish containing the medium was placed on the workbench, and incubated at $30{ }^{\circ} \mathrm{C}$ for $5 \mathrm{~d}$. All reagents used in the current study were purchased from Shanghai Aladin Biochemical Technology Co. LTD.

\subsection{Purification and further screening}

After a 5-d static cultivation, several distinct colonies with different morphological features were observed growing on the plate. The microbes were purified by passaging on CMC-Na medium until uniform colony morphology was obtained. Single-colony diameter was measured, and the plates were stained with a solution of Congo red $(1 \mathrm{~mL} \mathrm{mg}$ $\left.{ }^{1}\right)$ and de-stained with sodium chloride $\left(1 \mathrm{~mol} \mathrm{~L}^{-1}\right)$ to compare the cellulose degrading capacity of the isolates (Teather and Wood, 1982). The isolate degradation capacity was compared by calculating the ratio of the transparent halo around the colony to the colony diameter. A fungal isolate (strain WIU-01) with pronounced cellulose degrading ability was selected for detailed characterization.

\subsection{Isolate identification}

The fungal Internal Transcribed Spacer (ITS) rDNA gene was sequenced after PCR amplification using the ITS1 forward primer (5'-TCCGTAGGTGAACCTGCGG-3') and the ITS4 reverse primer (5'-TCCTCCGCTTATTGATATGC-3') (Schoch et al., 2012) at Shanghai SANGON Biotech Co., Ltd. The obtained sequence was then compared with the sequences deposited at (https://blast.ncbi.nlm.nih.gov/ Blast.cgi) using the Basic Local Alignment Search Tool. Strain WIU-01 was identified based on the degree of sequence homology (Embley and Stackebrandt, 1994). The phylogenetic tree was constructed by using MEGA v. 7 and the neighbor-joining method (bootstrap analysis with 500 replicates) (Tamura et al., 2004). ClustalW program was used for multiple-sequence alignment and the maximum composite likelihood method was used to compute evolutionary distances.

\subsection{EG activity and reducing sugar (RS) content determinations}

Logarithmic-phase fungal culture was refreshed by dilution $(1 \%, v / v)$ in $50 \mathrm{~mL}$ of fresh medium. EG activity was monitored in CMC-Na medium containing 1\% (w/v) CMC$\mathrm{Na}$ (prior to the experiment, CMC-Na was dissolved in acetate-sodium acetate buffer) as the substrate, as described by Ghose (1987), with slight modification. The experiment was conducted for $14 \mathrm{~d}$, with shaking (180 rpm), at $30^{\circ} \mathrm{C}$.

For the EG activity assay, the culture was sampled every 24 h. At each time point, $2 \mathrm{~mL}$ of culture were withdrawn and centrifuged at $4000 \mathrm{rpm}$ for $20 \mathrm{~min}$. Then, $0.5 \mathrm{~mL}$ of the supernatant was mixed with $1.5 \mathrm{~mL}$ of substrate in a $25-\mathrm{mL}$ volumetric flask, and incubated at $50{ }^{\circ} \mathrm{C}$ for $30 \mathrm{~min}$. Next, $1.5 \mathrm{~mL}$ of 3,5-dinitrosalicylic acid was added to stop the reaction (Miller, 1959). The samples were then heated in a boiling water bath for $5 \mathrm{~min}$ to allow color development. After cooling to room temperature, the volume was adjusted to the 25- $\mathrm{mL}$ mark with deionized water. Finally, sample absorbance was determined at $540 \mathrm{~nm}$ using an 
UV-vis spectrophotometer (UV-1800, Shimadzu, Kyoto, Japan). The amount of RS released was calculated using a curve constructed with glucose as a standard. The glucose standard curve was prepared as described elsewhere Ghose (1987). Inactivated sample (supernatant placed in a boiling water bath for $10 \mathrm{~min}$ ) was used as the blank control. One unit (U) of EG activity was defined as the amount of enzyme required for the release of $1 \mu \mathrm{mol}$ of glucose per min under the above conditions. The RS content was determined by DNS method.

\subsection{Fungal decomposition of submerged and emergent plant material}

The emergent plant Canna indica L. and submerged plant Hydrilla verticillata (L. f.) Royle were chosen for the hydrolysis assays as they are widely distributed in the tropical to temperate regions of Eurasia (Biswabijayinee et al., 2008, Cook, 1985).

Mature and healthy leaves and stems of $C$. indica and $H$. verticillata were used. The plant material was washed several times in water, heated at $100{ }^{\circ} \mathrm{C}$ for $30 \mathrm{~min}$, and dried at $80^{\circ} \mathrm{C}$. It was then crushed and sieved through a 60 size mesh. Next, $1.00 \mathrm{~g}$ of plant material was weighed (starting weight, W1) and mixed with $30 \mathrm{~mL}$ of mineral salt medium, according to Mandels and Weber (1969), with slight modification. Specifically, the solution contained $\mathrm{K}_{2} \mathrm{HPO}_{4} \cdot 3 \mathrm{H}_{2} \mathrm{O}, \quad\left(\mathrm{NH}_{4}\right)_{2} \mathrm{SO}_{4}, \mathrm{MgSO}_{4} \cdot 7 \mathrm{H}_{2} \mathrm{O}, \mathrm{CaCl}_{2} \cdot 2 \mathrm{H}_{2} \mathrm{O}$, $\mathrm{FeSO}_{4} \cdot 7 \mathrm{H}_{2} \mathrm{O}, \mathrm{ZnCl}_{2}$, and $\mathrm{CoCl}_{2} \cdot 6 \mathrm{H}_{2} \mathrm{O}$. The mixtures were then sterilized at $121{ }^{\circ} \mathrm{C}$ for $30 \mathrm{~min}$. One milliliter of logarithmically growing fugal culture (prepared as described in section 2.4) was used to inoculate the solutions, in triplicate, and cultivated with shaking (180 rpm) at $30{ }^{\circ} \mathrm{C}$. The culture medium without the fungal inoculum was used as the control. The cultures were sampled on days $1,2,4,7,13,20$, and 30 . At each sampling point, $2 \mathrm{~mL}$ of culture were centrifuged (4000 rpm for 20 min), and EG activity and RS were determined in the supernatants, as described in section 2.4 . The pellets were washed three times with ultrapure water, dried at $100{ }^{\circ} \mathrm{C}$, and then weighed (final weight, W2). The degradation rate of plant material was calculated using the following formula:

$$
\text { Degradation rate }(\%)=[(\mathrm{W} 1-\mathrm{W} 2) / \mathrm{W} 1] \times 100,
$$

W1 is the starting weight of the plant material in the mineral salt medium. W2 is the final weight of plant material after $30 \mathrm{~d}$ of incubation in the presence or absence of strain WIU-01, as described above.

Protein content in the samples was determined by using Bradford assay, with bovine serum albumin as the standard (Bradford, 1976).

\subsection{Fiber analysis of plant material}

CEL, HC, neutral detergent fibrils (NDF), acid detergent lignin (ADL), acid detergent fibrils (ADF), and ash content in the $C$. indica and $H$. verticillata material were determined by using Van Soest method (Van Soest et al., 1991).

\subsection{Statistical analysis}

Preliminary data analysis was performed using Excel 2016 (Microsoft Office, Microsoft). The figures and histograms were prepared using Origin Pro 2021 Beta3 (Origin Lab Corporation). Phylogenetic analyses were performed using MEGA v. 7. SPSS 22 (SPSS Statistics, IBM) was used for statistical analysis of independent samples ( $t$-test). Correlation analysis was performed based on Pearson correlation. The significance threshold was set at $p<0.05$ $\left({ }^{*} p<0.05, * * p<0.01, * * * p<0.001\right)$.

\section{Results and discussion}

\subsection{Morphological characterization and genetic} identification of the isolated fungus

In the current study, we aimed to isolate air-borne cellulolytic microbes. Based on the colony characteristics, microscopic morphological evaluation, and molecular and phylogenic analysis, we identified strain WIU-01 as a cellulolytic fungus. According to the microscopic evaluation (Figure $1 \mathrm{a}-\mathrm{c}$ ), the fungus grew on CMC-Na agar medium, forming an intricate network of extended hyphae. It formed fluffy and white colonies on CMC-Na agar medium at the early cultivation stage, which then became green, and finally, dark green. The conidia grew on the hyphae, with smooth stipes formed. The conidial heads were loose and radiated. The conidiophores were spherical, approximately $3.5 \mu \mathrm{m}$ in diameter, light green, and with small surface spines observed under $10 \times 100$ magnification. Based on the ITS rDNA sequence and phylogenic analysis, the fungus WIU-01 was identified as Aspergillus sydowii (Figure 1d) (Jurjevic et al., 2012).

Fungi are a large and diverse group of organisms. Filamentous fungi are preferentially used in commercial applications because they produce more enzymes than bacteria (Mrudula and Murugammal, 2011). Filamentous fungi from the genus Aspergillus are widely distributed in the biosphere and have a strong adaptive capacity (de Vries and Visser, 2001). Qaisar et al. (2014) have enriched an A. versicolor strain from paper reprocessing areas and sugarcane field, and showed its ability to degrade crystalline cellulose. Recent studies have shown that some A. sydowii strains hydrolyze CEL. For example, Ghareib et al. (1992) have studied the effect of alkali pretreatment on degradation of some cellulosic material (such as pods of bean, rice plant straw, wheat bran) by $A$. sydowii. Nair et al. (2008) isolated and purified xylanase from culture filtrate of $A$. sydowii with the wheat bran and birch wood as the carbon source. We here successfully isolated another $A$. sydowii strain, which utilizes CMC-Na as a single carbon source. We next proceeded to evaluate its EG and plantdegrading abilities in detail.

\subsection{EG activity and RS generation by isolate WIU-01}

We monitored its EG activity and RS levels in a medium containing $1 \%(\mathrm{w} / \mathrm{v}) \mathrm{CMC}-\mathrm{Na}$ to better characterize the crystalline CEL degrading activity of isolate WIU-01. RS content is indicative of the cellulolytic fungal capacity to utilize and decompose crystalline CEL and amorphous polysaccharides, with enzymes, such as EG, attacking the 
amorphous zone of CEL chains and releasing oligosaccharides of various lengths (Kaur et al., 2014). The experiment was performed over a 14-d period (Figure 2). Overall, the EG activity was low during the first $4 \mathrm{~d}$ of growth and then slowly increased over the remaining cultivation time. The EG activity reached a maximum $(0.027$ $U \mathrm{~mL}^{-1}$ ) at the end of the incubation period (day 14). The RS concentration trend was similarly to that of EG activity. Specifically, the RS concentration was low in the first $3 d$ of cultivation, before slowly rising on day 4 , and reaching the highest value $\left(0.41 \mathrm{mg} \mathrm{mL}^{-1}\right)$ at the end of the incubation period.
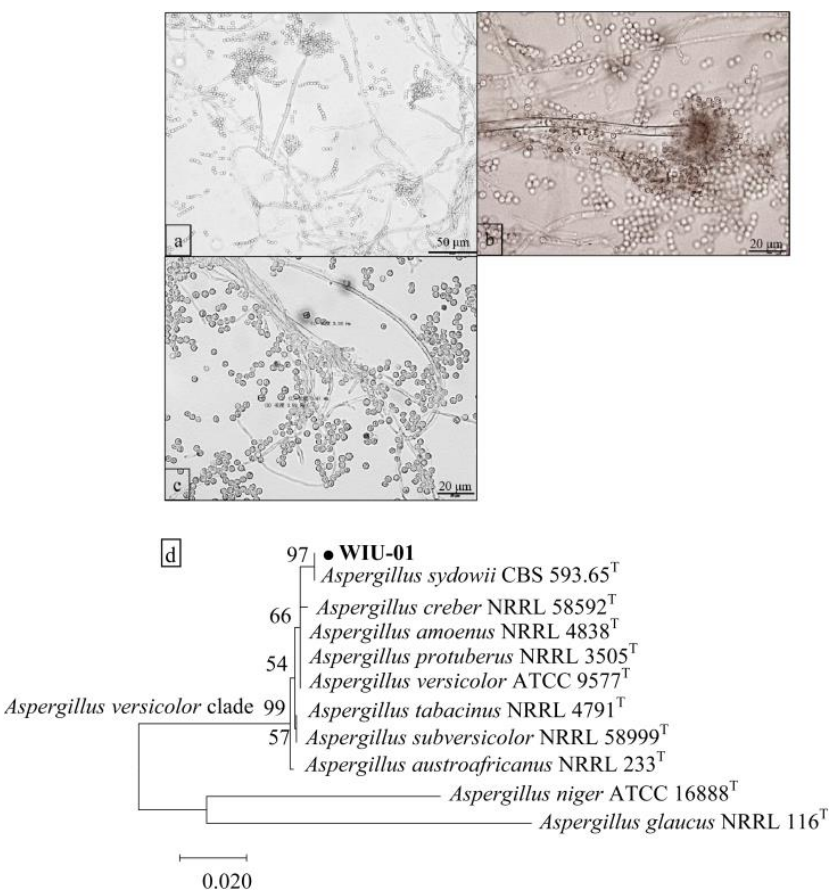

Figure 1. The morphology and phylogenic analysis of the cellulolytic fungal isolate WIU-01. (a) Representative fungal hypha viewed under $10 \times 40$ magnification. (b) Representative hypha viewed under $10 \times 100$ magnification. (c) Conidiophores viewed under $10 \times 100$ magnification. (d) Phylogenic analysis of the ITS rDNA gene sequence of the WIU-01 strain. The strain was identified as Aspergillus sydowii. The scale bar represents 0.2 substitutions per site, and the numbers at the nodes are bootstrap values (\%)

Fungi play a vital role in biomass degradation in nature but their EG activities vary widely. For instance, Mohapatra et al. (2018a) isolated an Aspergillus fumigatus strain from a soil-enriched decomposed lignocellulosic waste, and reported EG activity of $0.287 \mathrm{U} \mathrm{mL}^{-1}$ at $30{ }^{\circ} \mathrm{C}$. Yamada et al. (2014) studied a wild-type Aspergillus oryzae strain and reported EG activity of $0.0014 \mathrm{U} \mathrm{mL}^{-1}$. In that study, EG activity of the cellulase-expressing strains was $0.0299 \mathrm{U}$ $\mathrm{mL}^{-1}$. Further, Facchini et al. (2011) reported EG activity of an Aspergillus japonicus strain isolated from farming regions of São Paulo State (Brazil) as $0.55 \mathrm{U} \mathrm{mL}^{-1}$, which was higher than that of isolate WIU-01 reported in the current study. The EG activity of yet another $A$. versicolor strain, screened from soil samples from paper reprocessing areas and sugarcane field, was high, at approximately $200 \mathrm{U} \mathrm{mL}^{-}$
1 in 1\% CMC-Na culture (Qaisar et al., 2014). These discrepancies may be associated with individual strain characteristics and different testing conditions.

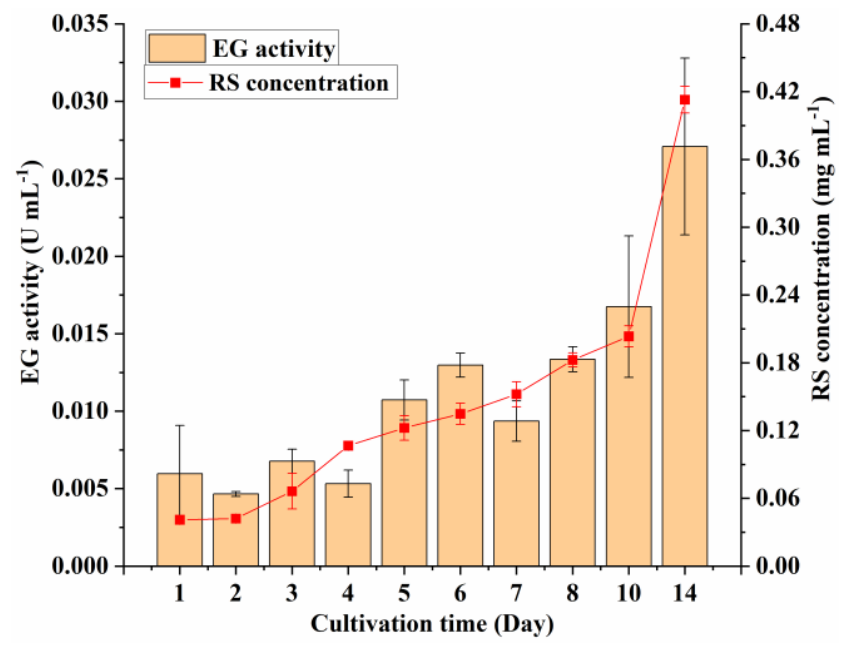

Figure 2. Endoglucanase (EG) activity of isolate WIU-01 and reducing sugar (RS) concentration during incubation in a medium containing $1 \%(\mathrm{w} / \mathrm{v}) \mathrm{CMC}-\mathrm{Na}$. The experiment was performed in triplicate and the data are presented as the mean \pm SD

\subsection{Degradation of emergent plant and submerged plant material by isolate WIU-01}

We next tested the ability of the strain isolate WIU-01 to degrade the emergent plant and submerged plant materials. In the absence of the fungus, protein concentration in a medium supplemented with $H$. verticillata powder (approximately $0.24 \mathrm{mg} \mathrm{mL}^{-1}$ ) was significantly higher than that in a medium supplemented with $C$. indica powder (approximately $0.075 \mathrm{mg} \mathrm{mL}^{-1}$ ) (Figure 3a). Conversely, protein content in both media inoculated with isolate WIU-01 showed a decreasing trend in the first $4 \mathrm{~d}$, which was significantly lower than that in the medium without the fungus. Upon prolonged cultivation with the fungus, the protein content increased to 0.14 and $0.42 \mathrm{mg} \mathrm{mL}^{-1}$ in the $C$. indica culture and in the $H$. verticillata culture, respectively.

Isolate WIU-01 hydrolyzed both plant materials (Figure 3b). The degradation rates of $C$. indica and $H$. verticillata materials in the absence of the fungus were approximately $20 \%$ and $14 \%$, respectively, throughout the incubation period ( $30 \mathrm{~d}$ ). The plant material is poorly soluble in cold water, but some polysaccharides and oligomers can become partially soluble in hot water during the autoclaving process (Zhang et al., 2020). This may be one of the reasons for the apparent degradation of the plant material in the medium without the fungus. Upon the addition of the fungus, the degradation rate of $C$. indica did not significantly change in the first $4 \mathrm{~d}$ of culture, but exhibited a marked increasing trend from day 7 , reaching a maximum of $29.75 \%$ on day 20 . The degradation of $H$. verticillata powder was relatively slower, but showed a similar trend, i.e., it first declined (days 1-2) and then increased (18.95\%). These findings indicate that isolate WIU-01 degrades and utilizes $C$. indica more efficiently that H. verticillata. 


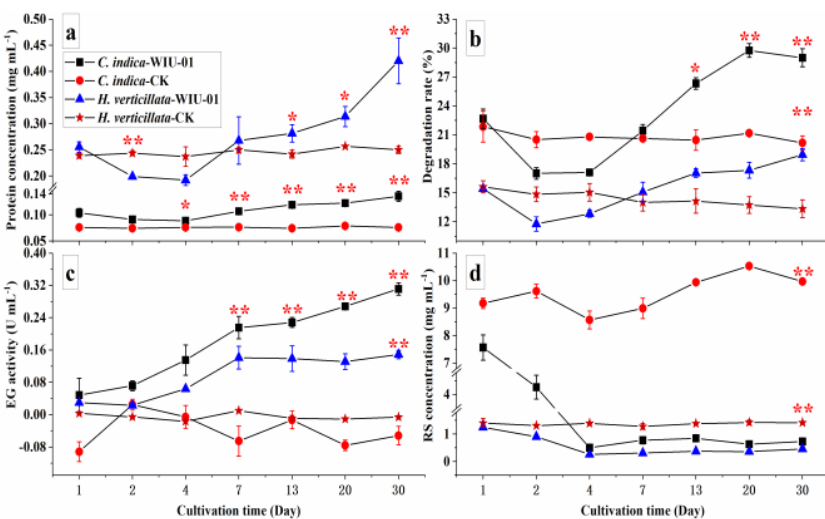

Figure 3. Changes in protein concentration, substrate degradation rate, endoglucanase (EG) activity, and reducing sugar (RS) concentration in media containing $C$. indica and $H$. verticillata powders, in the presence and absence of strain WIU-

01 . The cultures contained $C$. indica and $H$. verticillata plant material and were conducted at $30{ }^{\circ} \mathrm{C}$. (a) Protein concentration

in the culture medium. (b) The degradation rate of plant material. (c) EG activity of strain WIU-01. (d) RS concentration in the culture medium. Please note $y$-axis breaks in panels (a) and

(d). The experiment was performed in triplicate, and the data

are presented as the mean $\pm \mathrm{SD}\left({ }^{*} p<0.05,{ }^{*} p<0.01\right.$,

independent $t$-test, no-fungus control vs. fungus treatment)

EG activity of isolate WIU-01 in the plant materialsupplemented media gradually increased during the experiment (Figure 3c). Specifically, it increased from 0.049 to $0.31 \mathrm{U} \mathrm{mL}^{-1}$ with prolonged incubation in the $C$. indica culture, and it increased from 0.03 to $0.15 \mathrm{U} \mathrm{mL}^{-1}$ in the $H$. verticillata culture. The EG activity detected in the emergent and submerged plant media was thus significantly higher than that in the CMC-Na culture medium. This was consistent with the findings of Sun et al. (2008), who reported a higher enzyme activity of cellulolytic organisms exposed to complex substrates, such as wheat bran, than those grown in the presence of microcrystalline CEL substrate. Su et al. (2018) reported that EG activity was closely associated with the CEL degradation rate, which they attributed to the fact that enzymes break down the heterogeneous compounds to small components. The degradation rate of $C$. indica and $H$. verticillata exhibited an obviously increasing trend after day 4. Similarly, the EG activity rapidly increased phase day 2.

RS concentrations in the two plants materialsupplemented media in the absence of the fungus [i.e., generated during the sterilization stage of medium preparation $\left.\left(121{ }^{\circ} \mathrm{C}, 30 \mathrm{~min}\right)\right]$ were relatively stable, at approximately 9.54 and $1.37 \mathrm{mg} \mathrm{mL}^{-1}$ in the $C$. indica and $H$. verticillata media, respectively (Figure $3 d$ ). RS levels in the former were significantly higher than those in the latter. In the presence of the fungus, RS concentrations in the two media rapidly declined early in the cultivation (0-4 d), and then remained constant and low.

Detailed analysis of protein concentration, degradation rate, EG activity, and RS content revealed an interesting phenomenon: except for the EG activity, the values measured in the presence of isolate WIU-01 markedly dropped during the early adaptive stage of cultivation (days
0-4). This was followed by a rapid degradation phase between days 4 and 20, accompanied by a continuous increase in protein concentration and EG activity. However, the RS content remained low. This might be explained by the fungus initially adapting to the hydrophyte medium, and then rapidly proliferating and utilizing RS produced during medium sterilization. Low RS levels in the medium led to isolate WIU-01 breaking down and subsequently utilizing the fibril components of $C$. indica and $H$. verticillata. RS remained low during the remainder of cultivation, while the degradation rate and EG activity continued to increase. This might be because RS produced by isolate WIU-01 decomposing CEL only sufficed to maintain biomass growth, with no RS accumulation in the medium, or because microbes degraded and utilized CEL without the production of soluble RS (Gao et al., 2001).

The observed EG activity with $C$. indica and $H$. verticillata substrates was different. That could be explained by the different composition of the two substrates and was consistent with a reported differential EG activity levels in Aspergillus spp. incubated with different lignocellulosic substrates (Prajapati et al., 2020). In addition, the observed low EG activity during the late cultivation stage might be associated with the reduction of nutrient levels in the medium (Karim et al., 2015), as well as pH changes and accumulation of inhibitory metabolites (Abdullah et al., 2015). Further, enzyme binding may limit CEL accessibility to cellulase during the subsequent saccharification step (Qin et al., 2016). By contrast, some enzymes are adsorbed onto the CEL substrate, reducing the detectable cellulase activity in the medium filtrate (Zheng et al., 2020). Finally, formation of phenolic groups during the degradation process can hamper cellulolytic enzyme reaching CEL chains, thus influencing the measurable enzymatic activity (Mohapatra et al., 2017).

\subsection{Fiber fraction analysis of plant material and effect of} fungal activity

The plant cell wall mainly comprises $\mathrm{CEL}, \mathrm{HC}$, and lignin, which may inhibit the decomposition and utilization of plant biomass (Sánchez, 2009). Since we observed that isolate WIU-01 degraded $C$. indica and $H$. verticillata powder with different efficiency, we then analyzed the fiber fractions of the two plant materials, in the presence and absence of the fungus. $H$. verticillata powder contained significantly more $\mathrm{HC}, \mathrm{ADL}$, and ash than $C$. indica powder (Figure 4).

The analysis revealed that the $C$. indica and $H$. verticillata substrates had more CEL, NDF, and ADF than ADL, HC, and ash (Figure 4). In addition, CEL, NDF, and ADF levels in $C$. indica medium were significantly increased after the fungal treatment. By contrast, the fungal treatment significantly reduced the ash content in the medium. The fungal treatment significantly increased the NDF, ADF, and ash content in the $H$. verticillata medium. We observed no significant effect of the fungus on the ADL and $\mathrm{HC}$ content in the two media. 


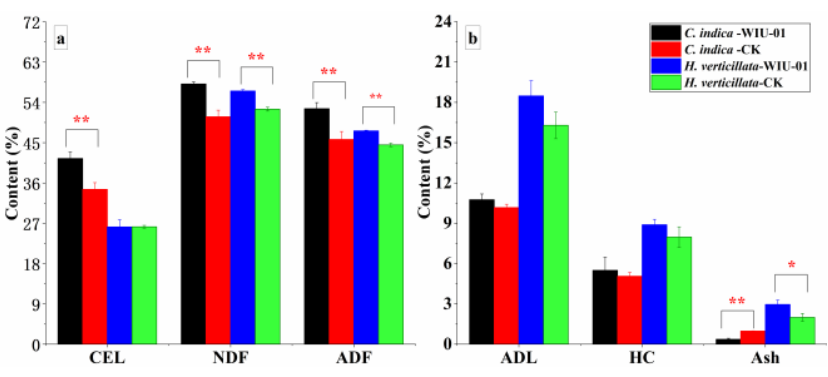

Figure 4. Fiber fractions in the $C$. indica (a) and $H$. verticillata (b) residual powders. Cellulose (CEL), hemicellulose $(H C)$, neutral detergent fibrils (NDF), acid detergent lignin (ADL), acid detergent fibrils (ADF), and ash levels were determined, with and without fungal treatment, after $30 \mathrm{~d}$ at $30^{\circ} \mathrm{C}$. The experiment was performed in triplicate, and the data are presented as the mean $+\mathrm{SD}\left({ }^{*} p<0.05,{ }^{* *} p<0.01\right.$, independent $t$-test, no-fungus control vs. fungus treatment)

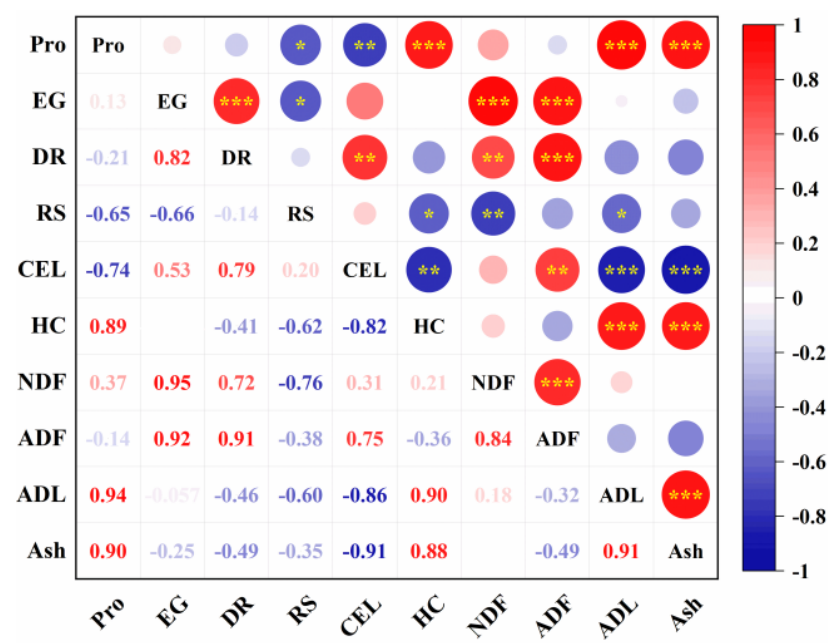

Figure 5. Correlation analysis of protein (Pro), degradation rate (DR), and fiber fractions (defined as in Figure 4 legend) in $C$. indica and $H$. verticillata residual powders with endoglucanase (EG) activity of isolate WIU-01. Pearson's correlation coefficient $(r)$ is shown. The magnitude of correlation is illustrated by the numbers (left) and circles (right), with the color scale indicated

$$
(* p<0.05, * * p<0.01, * * * p<0.001)
$$

We next performed a correlation analysis to investigate the factors that affected the WIU-01 EG activity in the media tested. We analyzed the relationship between EG activity, and the degradation rate and fibril components in the two plants (Figure 5). The analysis revealed a significant positive relationship between EG activity and degradation rate $(r=$ $\left.0.823^{* *}\right), \operatorname{NDF}\left(r=0.954^{* *}\right)$, and $\operatorname{ADF}\left(r=0.916^{* *}\right)$, with no significant correlation with the other factors examined. Similarly, degradation rate was significantly positively correlated with NDF $\left(r=0.715^{* *}\right), \operatorname{ADF}\left(r=0.912^{* *}\right)$, and CEL $\left(r=0.787^{* *}\right)$.

Lignin is found in all vascular plants and is a highly complex aromatic polymer, with a three-dimensional structure formed by benzene-propane units that are linked via ether and carbon-carbon bonds. On the other hand, HC is a branched polymer that is embedded in CEL elementary fibrils, with different sugars linked via glycosidic bonds (Jia et al., 2017). Generally, the main components of fibrils are determined using the Van Soest method (Van Soest et al., 1991), and their content is then calculated based on the dissolution degree of fibril components in a detergent solution. NDF represents the material with the protein, fat, and other extracts removed, mainly composed of CEL, HC, lignin, and ash. ADF mainly contains CEL, lignin, and ash (Wang et al., 2019). Fatica et al. (2019) reported that CEL (ADF minus $A D L$ ) and lignin ( $A D L$ ) contents have different effects on anaerobic ecosystems. Our analysis revealed a significant increase in CEL, NDF, and ADF in the $C$. indica fibrils and $H$. verticillata fibrils after enzymatic hydrolysis by the fungus.

\section{Conclusions}

We here successfully isolated and identified a cellulolytic Aspergillus sydowii isolate, strain WIU-01, from the air. The fungus exhibited a high activity with powdered material from the emergent plant $C$. indica (degradation rate of $29.75 \%$ and EG activity of $0.31 \mathrm{U} \mathrm{mL}^{-1}$ ) indicating the possibility of its application in biomass decomposition and utilization. However, it showed a low activity with powdered material from the submerged plant $H$. verticillata (degradation rate of $21.95 \%$ and EG activity of $0.16 \mathrm{U} \mathrm{mL} \mathrm{m}^{-1}$ ). The CMCase activity significantly impacted the degradation rate. Further, correlation analysis indicated that CEL, HC, ADL, and ash in the hydrophyte residue significantly affected fungal biomass growth. Overall, these findings indicate that some air-borne fungi potentially utilize CEL, and may play a role in large-scale macrophyte degradation without the need for acid-base pretreatment. However, there are some limitations to the immediate use of the novel $A$. sydowii isolate, e.g., its EG activity is relatively low and the fungus may produce some toxic secondary metabolites. Further studies could focus on, e.g., extracting the EG gene encoded by strain WIU-01 and generation of recombinant proteins for specific applications. Nonetheless, the current study underpins the cellulolytic potential of naturally occurring microorganisms and the need for their continued characterization.

\section{Funding}

This research was funded by the Strategic Priority Research Program of the Chinese Academy of Sciences, grant number XDA23040401, and the State Key Laboratory of Freshwater Ecology and Biotechnology, grant number 2019FBZ03.

\section{Acknowledgments}

The authors would like to thank Liping Zhang for organizing the sampling survey, and our colleagues from State Key Laboratory of Freshwater Ecology and Biotechnology for collecting and identifying hydrophytes.

\section{Data availability}

All data related to this study are presented in this article.

\section{Conflicts of interest}

The authors declare no conflict of interest. 


\section{References}

Abdullah R., Zafar W., Nadeem M., Iqtedar M., Naz S., Syed Q. and Kaleem A. (2015). Process optimisation for the biosynthesis of cellulase by Bacillus PC-BC6 and its mutant derivative Bacillus N3 using submerged fermentation. Natural Product Research, 29, 1133-1138.

Amezcua-Allieri M.A., Sánchez Durán T. and Aburto J. (2017). Study of chemical and enzymatic hydrolysis of cellulosic material to obtain fermentable sugars. Journal of Chemistry, 2017, 1-9.

Benoit I., Culleton H., Zhou M., Difalco M., Aguilar-Osorio G., Battaglia E., Bouzid O., Brouwer C., El-Bushari H.B.O., Coutinho P.M., Gruben B.S., Hilden K.S., Houbraken J., Barboza L.a.J., Levasseur A., Majoor E., Makela M.R., Narang H.M., Trejo-Aguilar B., Van Den Brink J., Vankuyk P.A., Wiebenga A., Mckie V., Mccleary B., Tsang A., Henrissat B. and De Vries R.P. (2015). Closely related fungi employ diverse enzymatic strategies to degrade plant biomass. Biotechnol Biofuels, 8, 107.

Bhat K.M. and Maheshwari R. (1987) Sporotrichum thermophile growth, cellulose degradation, and cellulase activity, Applied and Environmental Microbiology, 53, 2175-2182.

Biswabijayinee P., Acharya L., Mukherjee A., Panda M. and Panda P. (2008). Molecular characterization of ten cultivars of Canna lilies (Canna Linn.) using PCR based molecular markers (RAPDs and ISSRs). International Journal of Integrative Biology, 2, 129-137.

Bradford M.M. (1976). A rapid and sensitive method for the quantitation of microgram quantities of protein utilizing the principle of protein-dye binding. Analytical Biochemistry, 72, 248-254.

Cook C.D.K. (1985). Range extensions of aquatic vascular plant species. Journal of Aquatic Plant Management, 23, 1-6.

De Vries R.P. and Visser J. (2001). Aspergillus enzymes involved in degradation of plant cell wall polysaccharides. Microbiology and Molecular Biology Reviews, 65, 497-522, table of contents.

Embley T.M. and Stackebrandt E. (1994). The molecular phylogeny and systematics of the actinomycetes. Annual Review of Microbiology, 48, 257-289.

Facchini F.D.A., Vici A.C., Jorge J.O.A., Terenzi H.C.F., Polizeli M.D.L.T.D.M., Reis V.R.A. and Reis R.A. (2011). Production of fibrolytic enzymes by Aspergillus japonicus C03 using agroindustrial residues with potential application as additives in animal feed. Bioprocess and Biosystems Engineering, 34, 347355.

Fatica A., Di Lucia F., Marino S., Alvino A., Zuin M., De Feijter H., Brandt B., Tommasini S., Fantuz F. and Salimei E. (2019). Study on analytical characteristics of Nicotiana tabacum L., cV. Solaris biomass for potential uses in nutrition and biomethane production. Scientific Reports, 9.

Gao P.-J., Chen G.-J., Wang T.-H., Zhang Y.-S. and Liu J. (2001). Non-hydrolytic Disruption of Crystalline Structure of Cellulose by Cellulose Binding Domain and Linker Sequence of Cellobiohydrolase I from Penicillium janthinellum. Sheng wu hua xue yu sheng wu wu li xue bao Acta biochimica et biophysica Sinica, 33, 13-18.

Geary P.M. and Kim S.Y. (2001). The impact of biomass harvesting on phosphorus uptake by wetland plants. Water Science and Technology, 44, 61-67.
Ghareib M., Youssef K.A. and Nour El Dein M.M. (1992). Effect of alkali pretreatment on degradation of some cellulosic wastes by Aspergillus sydowii. Zentralblatt für Mikrobiologie, 147, 551-556.

Ghose T.K. (1987). Measurement of cellulase activities. Pure and Applied Chemistry, 59, 257-268.

Hernández M., Hernández-Coronado M.J., Montiel M.D., RodríGuez J., Pérez M.I., Bocchini P., Galletti G.C. and Arias M.E. (2001). Pyrolysis/gas chromatography/mass spectrometry as a useful technique to evaluate the ligninolytic action of streptomycetes on wheat straw. Journal of Analytical and Applied Pyrolysis, 58-59, 539-551.

Herve V., Le Roux X., Uroz S., Gelhaye E. and Frey-Klett P. (2014). Diversity and structure of bacterial communities associated with Phanerochaete chrysosporium during wood decay. Environmental Microbiology, 16, 2238-2252.

Jia J., Zhang W., Yang Z., Yang X., Wang N. and Yu X. (2017). Novel magnetic cross-linked cellulase aggregates with a potential application in lignocellulosic biomass bioconversion. Molecules, 22.

Jurjevic Z., Peterson S.W. and Horn B.W. (2012). Aspergillus section Versicolores: nine new species and multilocus DNA sequence based phylogeny. IMA Fungus, 3, 59-79.

Karim A., Nawaz M.A., Aman A. and UI Qader S.A. (2015). Hyper production of cellulose degrading endo $(1,4) \beta$-d-glucanase from Bacillus licheniformis KIBGE-IB2. Journal of Radiation Research and Applied Sciences, 8, 160-165.

Kaur B., Oberoi H.S. and Chadha B.S. (2014). Enhanced cellulase producing mutants developed from heterokaryotic Aspergillus strain. Bioresource Technology, 156, 100-107.

Kowalczyk J.E., Lubbers R.J.M., Peng M., Battaglia E., Visser J. and De Vries R.P. (2017). Combinatorial control of gene expression in Aspergillus niger grown on sugar beet pectin. Scientific Reports, 7, 12356.

Makela M.R., Difalco M., Mcdonnell E., Nguyen T.T.M., Wiebenga A., Hilden K., Peng M., Grigoriev I.V., Tsang A. and and De Vries R.P. (2018). Genomic and exoproteomic diversity in plant biomass degradation approaches among Aspergilli. Studies in Mycology, 91, 79-99.

Mandels M. and Weber J. (1969). The Production of Cellulases. Cellulases and Their Applications.

Miller G.L. (1959). Use of Dinitrosalicylic Acid Reagent for Determination of Reducing Sugar. Analytical Chemistry, 31, 426-428.

Mohapatra S., Mishra C., Behera S.S. and Thatoi H. (2017). Application of pretreatment, fermentation and molecular techniques for enhancing bioethanol production from grass biomass - A review. Renewable and Sustainable Energy Reviews, 78, 1007-1032.

Mohapatra S., Padhy S., Das Mohapatra P.K. and Thatoi H.N. (2018a). Enhanced reducing sugar production by saccharification of lignocellulosic biomass, Pennisetum species through cellulase from a newly isolated Aspergillus fumigatus. Bioresource Technology, 253, 262-272.

Mohapatra S., Padhy S., Das Mohapatra P.K. and Thatoi H.N. (2018b). Enhanced reducing sugar production by saccharification of lignocellulosic biomass, Pennisetum species through cellulase from a newly isolated Aspergillus fumigatus. Bioresource Technology, 253, 262-272.

Mrudula S. and Murugammal R. (2011). Production of cellulase by Aspergillus niger under submerged and solid state 
fermentation using coir waste as a substrate. Brazilian Journal of Microbiology, $\mathbf{4 2 .}$.

Nair S.G., Sindhu R. and Shashidhar S. (2008). Purification and biochemical characterization of two xylanases from Aspergillus sydowii SBS 45. Applied Biochemistry and Biotechnology, 149, 229-243.

Prajapati B.P., Jana U.K., Suryawanshi R.K. and Kango N. (2020). Sugarcane bagasse saccharification using Aspergillus tubingensis enzymatic cocktail for $2 \mathrm{G}$ bio-ethanol production. Renewable Energy, 152, 653-663.

Qaisar S., Zohra R.R., Aman A. and and Qader S.A. (2014). Enhanced production of cellulose degrading CMCase by newly isolated strain of Aspergillus versicolor. Carbohydrate Polymers, 104, 199-203.

Qin L., Li W.C., Liu L., Zhu J.Q., Li X., Li B.Z. and Yuan Y.J. (2016). Inhibition of lignin-derived phenolic compounds to cellulase. Biotechnology for Biofuels, 9.

Raulo R., Kokolski M. and Archer D.B. (2016). The roles of the zinc finger transcription factors $X \ln R, C \operatorname{lr} A$ and $C l r B$ in the breakdown of lignocellulose by Aspergillus niger. $A M B$ Express, 6, 5.

Sajab M.S., Mohan D., Santanaraj J., Chia C.H., Kaco H., Harun S. and Kamarudin N.H.N. (2019). Telescopic synthesis of cellulose nanofibrils with a stable dispersion of $\mathrm{Fe}(0)$ nanoparticles for synergistic removal of 5-fluorouracil. Scientific Reports, 9, 11703.

Sánchez C. (2009). Lignocellulosic residues: biodegradation and bioconversion by fungi. Biotechnology Advances, 27, 185194.

Schoch C.L., Seifert K.A., Huhndorf S., Robert V., Spouge J.L., Levesque C.A., Chen W., Fungal Barcoding C. and Fungal Barcoding Consortium Author L. (2012). Nuclear ribosomal internal transcribed spacer (ITS) region as a universal DNA barcode marker for Fungi. Proceedings of the National Academy of Sciences of the United States of America, 109, 6241-6246.

Su Y., Yu X., Sun Y., Wang G., Chen H. and Chen G. (2018). Evaluation of screened lignin-degrading fungi for the biological pretreatment of corn stover. Scientific Reports, 8, 5385.

Sun X., Liu Z., Qu Y. and Li X. (2008). The effects of wheat bran composition on the production of biomass-hydrolyzing enzymes by Penicillium decumbens. Applied Biochemistry and Biotechnology, 146, 119-128.

Tamura K., Nei M. and Kumar S. (2004). Prospects for inferring very large phylogenies by using the neighbor-joining method. Proceedings of the National Academy of Sciences of the United States of America, 101, 11030-11035.

Teather R.M. and Wood P.J. (1982). Use of congo redpolysaccharide interactions in enumeration and characterization of cellulolytic bacteria from the bovine rumen. Applied and Environmental Microbiology, 43, 777780.

Van Echelpoel W. and Goethals P.L.M. (2018). Variable importance for sustaining macrophyte presence via random forests: data imputation and model settings. Scientific Reports, 8, 14557.

Van Soest P.J., Robertson J.B. and Lewis B.A. (1991) Methods for dietary fiber, neutral detergent fiber, and nonstarch polysaccharides in relation to animal nutrition, Journal of Dairy Science, 74, 3583-3597.
Wang F., Zhang D., Chen M., Yi W. and Wang L. (2019). Characteristics of corn stover components pyrolysis at low temperature based on detergent fibers. Frontiers in Bioengineering and Biotechnology, 7, 188.

Welsch M. and Yavitt J.B. (2003). Early stages of decay of Lythrum salicaria L. and Typha latifolia L. in a standing-dead position. Aquatic Botany, 75, 45-57.

Yamada R., Yoshie T., Wakai S., Asai-Nakashima N., Okazaki F., Ogino C., Hisada H., Tsutsumi H., Hata Y. and Kondo A. (2014). Aspergillus oryzae-based cell factory for direct kojic acid production from cellulose. Microbial Cell Factories, 13, 71.

Yoon J.-J. and Kim Y.-K. (2005). Degradation of crystalline cellulose by the brown-rot basidiomycete fomitopsis palustris. Journal of Microbiology, 43, 487-492.

Zhang W.J., Wang S., Kang C.Z., Lv C.G., Zhou L., Huang L.Q. and Guo L.P. (2020). Pharmacodynamic material basis of traditional Chinese medicine based on biomacromolecules: a review. Plant Methods, 16, 26.

Zhao F., Yang W., Zeng Z., Li H., Yang X., He Z., Gu B., Rafiq M.T. and Peng H. (2012). Nutrient removal efficiency and biomass production of different bioenergy plants in hypereutrophic water. Biomass and Bioenergy, 42, 212-218.

Zheng W., Lan T., Li H., Yue G. and Zhou H. (2020). Exploring why sodium lignosulfonate influenced enzymatic hydrolysis efficiency of cellulose from the perspective of substrateenzyme adsorption. Biotechnology for Biofuels, 13, 19. 